The AAPS Journal 2006; 8 (3) Article 66 (http://www.aapsj.org).

Themed Issue: Proceedings of the 2005 AAPS Biotec Open Forum on Aggregation of Protein Therapeutics

Guest Editor - Steve Shire

\title{
Protein Aggregation and Bioprocessing
}

Submitted: February 23, 2006; Accepted: June 22, 2006; Published: September 15, 2006

Mary E.M. Cromwell, ${ }^{1}$ Eric Hilario, ${ }^{1}$ and Fred Jacobson ${ }^{2}$

${ }^{1}$ Early Stage Pharmaceutical Development, Genentech Inc, South San Francisco, CA

${ }^{2}$ Early Stage Analytical Development, Genentech Inc, South San Francisco, CA

\section{ABSTRACT}

Protein aggregation is a common issue encountered during manufacture of biotherapeutics. It is possible to influence the amount of aggregate produced during the cell culture and purification process by carefully controlling the environment (eg, media components) and implementing appropriate strategies to minimize the extent of aggregation. Steps to remove aggregates have been successfully used at a manufacturing scale. Care should be taken when developing a process to monitor the compatibility of the equipment and process with the protein to ensure that potential aggregation is minimized.

KEYWORDS: Aggregation, self-association, cell culture, purification, filling, manufacture

\section{INTRODUCTION}

This article is based on a presentation given at the 2005 AAPS Open Forum on Protein Aggregation in San Francisco, CA on June 5, 2006, and briefly describes some anecdotal encounters with aggregation during the manufacturing process. Little information has been published on the extent and causes of aggregation during bioprocessing for pharmaceutical proteins. Conversations with colleagues at conferences such as this Open Forum reveal that the observation of aggregation during manufacture is not uncommon. This article serves to acknowledge that there are challenges with aggregation during the manufacturing process and to briefly review some of the approaches taken to minimize the aggregates.

It should be noted that many proteins require association to be active, and that the associated state is the native form for those proteins. The issues with aggregation referred to in this article do not pertain to these native forms, but rather focus on the proteins where multimeric forms are undesirable.

Corresponding Author: Mary E.M. Cromwell, MS 96A, 1 DNA Way, Genentech Inc, South San Francisco, CA 94080. Tel: (650) 225-1955; Fax: (650) 225-7234;

E-mail: cromwell@gene.com

\section{What Is Protein Aggregation?}

Aggregation is a general term that encompasses several types of interactions or characteristics. Aggregates of proteins may arise from several mechanisms and may be classified in numerous ways, including soluble/insoluble, covalent/noncovalent, reversible/irreversible, and native/ denatured. For protein therapeutics, the presence of aggregates of any type is typically considered to be undesirable because of the concern that the aggregates may lead to an immunogenic reaction (small aggregates) or may cause adverse events on administration (particulates).

There is no consistent definition of what is meant by a "soluble" aggregate, so working definitions are often employed. For the purpose of this article, soluble aggregates refer to those that are not visible as discrete particles and that may not be removed by a filter with a pore size of $0.22 \mu \mathrm{m}$. Conversely, insoluble aggregates may be removed by filtration and are often visible to the unaided eye. Both types of aggregates may be problematic for the development of a therapeutic protein. There are clear guidelines and limitations on the number of particles $\geq 10$ $\mu \mathrm{m}$ and $\geq 25 \mu \mathrm{m}$ in size that may be present in pharmaceutical preparations. ${ }^{1}$ However, the levels of soluble aggregates such as dimers and trimers that are acceptable are not well defined.

Covalent aggregates arise from the formation of a chemical bond between 2 or more monomers. Disulfide bond formation resulting from previously unpaired free thiols is a common mechanism for covalent aggregation. ${ }^{2,3}$ Oxidation of tyrosines may also result in covalent aggregation through the formation of bityrosine. ${ }^{4,5}$ For some proteins, a covalent interaction between monomers is required to form a stable protein structure. Many of the growth factors, including Vascular Endothelial Growth Factor (VEGF), Transforming Growth Factor- $\beta$ 1, and Nerve Growth Factor, have extremely stable structures owing to the presence of several disulfide bonds, including one that exists between monomers to lead to a native covalent dimer.

Reversible protein aggregation typically results from relatively weak noncovalent protein interactions. The reversibility is sometimes indicative of the presence of equilibrium between the monomer and higher order forms. This equilibrium may 
shift as a result of a change in solution conditions such as a decrease in protein concentration or a change in $\mathrm{pH}$. A weak, reversible self-association of this type has been observed in a monoclonal antibody to VEGF. ${ }^{6}$ On occasion, reversible protein self-association manifests itself as an increase in viscosity. ${ }^{7}$

The effect of the presence of self-associated species is not always known. Both the thermodynamics and the kinetics of the system may assist in understanding how to control the association of the protein. In addition, this knowledge aids in determining how serious the presence of associated species may be during the development of a protein therapeutic. Both the potential for increased exposure to the associated species and the route of administration present potential safety concerns. In vivo, it is not unusual for aggregates to clear more slowly than their monomeric counterparts, leading to increased circulating levels of and exposure to the associated species. For covalently aggregated insulin, the half-life for clearance of aggregates was measured to be approximately double that observed for monomer in a study in human volunteers, and the authors attributed the fairly high immunogenicity response to these preparations to the aggregate species. ${ }^{8}$ However, there may be some cases in which the dissociation of the aggregate forms is much faster than the clearance rate. For example, if dissociation occurs rapidly on dilution, the presence of reversible dimers may not be troublesome if delivered intravenously. Conversely, slowly dissociating dimers or other aggregate species administered subcutaneously may trigger an immunogenic response. ${ }^{9}$

Historically, investigators believed that denaturation was a prerequisite for protein aggregation. Exposure of hydrophobic surfaces upon denaturation results in favorable protein: protein interactions in aqueous solutions. It is true that this type of interaction leads to the formation of aggregates in many proteins and may cause extreme precipitation. However, the role of native protein interactions in the formation of self-associated species has recently become more appreciated. Small perturbations in protein structure may expose hydrophobic surfaces that lead to aggregation. Electrostatic interactions have been implicated in the formation of self-associated species of a monoclonal antibody, ${ }^{7}$ while dipole-dipole interactions are believed to be the cause of fibrillogenic association of $\beta$-sheets. ${ }^{10}$

Just as there are many types of interactions that can lead to protein aggregation, there are many environmental factors that can lead to aggregation. ${ }^{11}$ Solution conditions such as temperature, protein concentration, $\mathrm{pH}$, and the ionic strength may affect the amount of aggregate observed. The presence of certain ligands, including specific ions, may enhance aggregation. Stresses to the protein such as freezing, exposure to air, or interactions with metal surfaces may result in surface denaturation, which then leads to the for- mation of aggregates. Finally, mechanical stresses may cause protein aggregation. Each of these environmental factors is typically encountered during bioprocessing.

During the manufacture of protein therapeutics, the protein is exposed to many stresses. Take, for example, the typical production of a monoclonal antibody from a mammalian cell culture. During the cell culture, the protein is secreted from the cell into the medium containing the cells, ions, nutrients for the cells, host cell proteins (including proteases), dissolved oxygen, and other species. This cellular suspension at near neutral $\mathrm{pH}$ is held at temperatures above $30^{\circ} \mathrm{C}$ for several days. Once a sufficient amount of protein has been made, the cell culture fluid is harvested and purified over Protein A chromatography. This affinity chromatography elutes the monoclonal antibody using an acidic solution. Polishing steps typically include cation exchange chromatography, which elutes the protein with high ionic strength solutions, and anion exchange chromatography, which employs high $\mathrm{pH}$ conditions to purify the monoclonal antibody from process-related impurities. Finally, the protein is formulated using ultrafiltration/diafiltration. The formulated protein may be stored frozen for some period of time before being filled into its final container. Throughout production, the protein solution is pumped, stirred, and filtered. The solution encounters containers made of different materials of composition including stainless steel, glass, and plastic. All of these processes can potentially result in the formation of aggregates.

\section{Aggregation in Cell Culture}

There are several opportunities for protein aggregation to occur during cell culture. During expression, accumulation of high amounts of protein may lead to intracellular aggregation owing to either the interactions of unfolded protein molecules or to inefficient recognition of the nascent peptide chain by molecular chaperones responsible for proper folding. ${ }^{12}$ As described above, secretion of the protein into the cell culture medium exposes the protein to conditions that may be unfavorable for protein stability. Judicious selection of the expression system and culture conditions is important to minimize aggregation.

An example of the effect of overexpression on protein aggregation can be found in the Escherichia coli production of the coxsackievirus and adenovirus receptor (CAR) amino-terminal immunoglobulin variable region domain, a protein fragment that is $\sim 16 \mathrm{kd} .{ }^{13}$ The original experiments with culture temperatures between $18^{\circ} \mathrm{C}$ and $37^{\circ} \mathrm{C}$ targeted expression in the periplasmic space, but undetectable levels of protein were observed. A 22-amino acid carboxy-terminal extension was added as a result of a vector change to target the cytoplasmic space. With culture conditions at $37^{\circ} \mathrm{C}$, expression was observed but the protein was found to be 
completely insoluble. Shifting the culture temperature to $18^{\circ} \mathrm{C}$ resulted in the expression of largely soluble protein with this 22-amino acid extension. Studies with this protein and several other fusion peptides concluded that the high negative charge of the peptide most likely prevented aggregation due to electrostatic repulsion, indirectly minimizing protein aggregation by allowing the protein to spend more time in the folding pathway. ${ }^{12}$

The choice of the components in the growth medium used during cell culture may affect the observed aggregation by influencing the ability of the protein to fold to a native structure. During protein production, disulfide bond formation occurs in the endoplasmic reticulum (ER) of cells. Proper disulfide bond formation is critical for folding of native protein structures. Typically an enzyme-catalyzed process, formation of the disulfide bond typically requires an oxidative environment. ${ }^{14}$ In the absence of this environment, the free thiols on the cysteines may remain unpaired, leading to improper folding.

It has been reported that monoclonal antibodies expressed in a Chinese hamster ovary (CHO) cell line sometimes contain unpaired thiols. ${ }^{15}$ In one study, the effect of addition of copper sulfate to the cell culture medium on the level of free thiol observed in a monoclonal antibody produced from $\mathrm{CHO}$ cells using this culture was examined. ${ }^{16} \mathrm{Cu}^{2+}$, a known oxidizing agent, was added to the medium to drive disulfide bond formation. The free thiol content of the Protein A-purified protein was assessed by hydrophobic interaction chromatography of a papain-digested preparation. The results were quantified as percentage of free thiol per Fab. Without $\mathrm{CuSO}_{4}$ in the medium, the level of free thiol was quantified as $37 \%$. Adding $\mathrm{CuSO}_{4}$ at concentrations of 5 , 50 , and $100 \mu \mathrm{mol} / \mathrm{L}$ decreased the free thiol content to $12 \%$, $3 \%$, and $3 \%$, respectively. These data show that addition of as little as $5 \mu \mathrm{mol} / \mathrm{L} \mathrm{CuSO}_{4}$ to the cell culture medium resulted in a 3 -fold reduction in the level of free thiol observed in the purified antibody with greater reduction observed when higher $\mathrm{CuSO}_{4}$ concentrations were used.

The presence of a free thiol may affect long-term stability of the protein. During development of a formulation for a monoclonal antibody, aggregates formed during storage of the lyophilized product at $30^{\circ} \mathrm{C} .{ }^{7}$ Sodium dodecyl sulfatepolyacrylamide gel electrophoresis (SDS-PAGE) was used to determine that these aggregates were covalent. The disappearance of these aggregates upon reduction indicated that they were the result of disulfide bond formation. Preparative size exclusion chromatography (SEC) was used to isolate fractions of monomer and dimer for characterization. The most striking difference between these fractions was the level of free thiol observed, with the monomer fraction showing $\sim 1 \mathrm{~mol}$ of free thiol per mole of protein, while the dimer showed $<0.1 \mathrm{~mol}$ free thiol per mole of protein. These results indicate that the free thiol on 1 monomer can interact with that on another monomer, leading to a covalent dimer form. Thus, elimination of this free thiol on the monomer should result in better lyophilized stability of the monomer.

Noncovalent aggregates have also been observed to form during cell culture. During clinical development of a monoclonal antibody, the Protein A pool showed an increase in aggregate content after modest changes were made to the cell culture process, including the use of an amplified cell line, a shift in the temperature of the culture, and subtle changes to the medium used for production. To understand the cause of the increased aggregation, a study was performed in which samples were taken from the cell culture fluid and purified over an analytical scale Protein A column. This Protein A purified sample was analyzed by SEC to determine the percentage aggregate in each sample. The results are displayed in Figure 1. Between days 3 and 13, there was an almost linear increase in the aggregate content. A complication in understanding these results is that there was accumulation of product in the cell culture medium during this time. This increase in protein concentration alone could cause the increase in aggregation observed. To determine if this was the case, at day 13 the cells were removed from the cell culture medium and the harvested cell culture fluid was incubated at $37^{\circ} \mathrm{C}$ for an additional 2 weeks. These results are represented by the dashed line in Figure 1. There was no change in protein concentration during this time; aggregation occurred at the same apparent rate as when the cells were present. The greater the length of

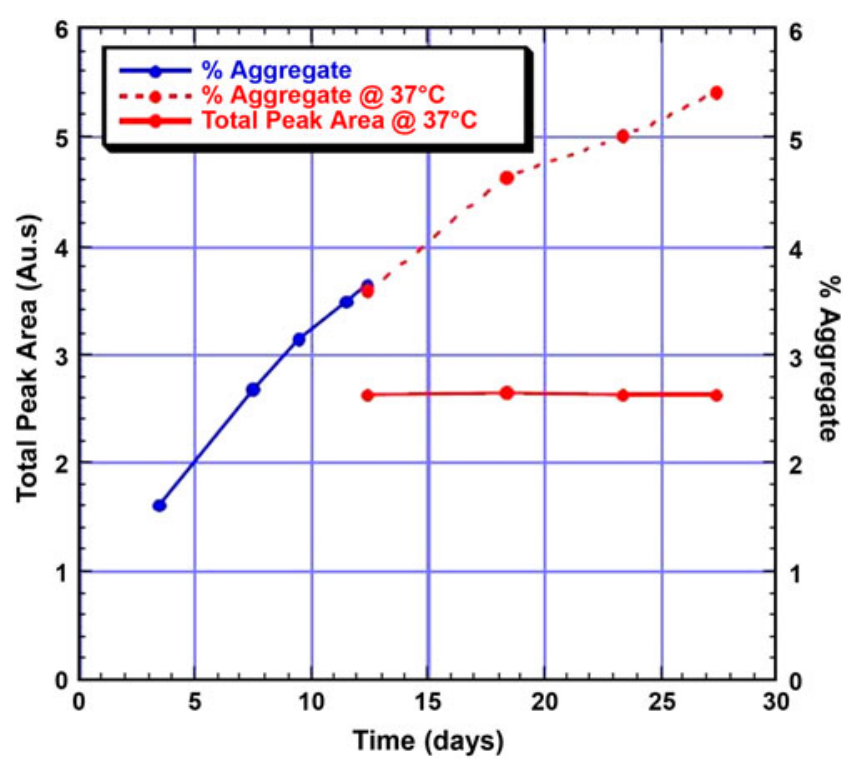

Figure 1. Increase in aggregate for a MAb in cell culture fluid. Samples were analyzed after Protein A purification. The solid line represents samples taken during cell culture. The dashed line indicates samples taken from the harvested cell culture solution held at $37^{\circ} \mathrm{C}$. The total peak area was constant for samples taken from the harvested cell culture solution and indicates that the protein concentration did not change during incubation. 
The AAPS Journal 2006; 8 (3) Article 66 (http://www.aapsj.org).

time the protein was held in the cell culture medium at elevated temperature, the greater the amount of aggregate observed. Thus, it would be possible to minimize the extent of aggregation in the purified product by limiting the time of the cell culture process.

\section{Aggregation During Purification}

Once a therapeutic protein has been produced from a cell culture (or other expression system), it is imperative that it be further purified to reduce or remove host cell proteins, viruses, and process-related impurities. To achieve the high level of purity required, multiple orthogonal purification techniques are employed. These techniques exploit differences in affinity, charge, size, or other properties of the desired protein from the impurities to enhance purification. As mentioned above, the result may be that the protein experiences a wide range of $\mathrm{pH}$, ionic strength, and protein concentrations during the process. Each condition experienced by the protein may affect the degree of aggregation observed.

Protein A affinity chromatography is a powerful purification technique used in the production of monoclonal antibodies. The binding of antibodies to protein A resin is highly specific. The high affinity of the monoclonal antibodies to the resin requires acidic conditions to elute the proteins from the resin. Exposure to these acidic conditions can result in the formation of aggregates. This has been observed in preparations of CamPath-1H (alemtuzumab), in which elution from the Protein A column with $0.1 \mathrm{M}$ sodium citrate, $\mathrm{pH}$ 3.2 , resulted in a pool containing protein with $\sim 25 \%$ aggregated material. ${ }^{17}$

The choice of the components of the elution buffer from Protein A columns can affect not only the recovery of the protein from the column but also the quality of the protein in the resulting pool. The recovery of a monoclonal antibody from Protein A using different buffer species in the elution was examined. ${ }^{18}$ This study demonstrated that the use of arginine in the elution buffer yielded a higher recovery with a lower percentage of aggregate in the recovered pool compared with the use of citrate, guanidine hydrochloride, and amino acids such as glycine and histidine. ${ }^{18}$ The pool eluted with guanidine hydrochloride showed good recovery, but the protein was extensively aggregated presumably from the denaturing effects of the guanidine.

If aggregates are created either during cell culture or during processing, it may be possible to remove them during the purification process by exploiting the differences in physical properties of the monomers and aggregates, including surface charge, hydrophobicity, and size. As described above, CamPath-1H contains $~ 25 \%$ aggregated species after Protein A purification. The purification process for this antibody uses preparative SEC to reduce the levels of aggregates in the final solution. By employing either Sephacryl 300 or Superdex 200, gel filtration resins that separate according to size, it is possible to achieve final pools with $<2 \%$ aggregate. ${ }^{17}$

Although it is technically possible to reduce the level of aggregates in a protein solution using SEC, it is often not cost efficient to do so. Preparative SEC is typically inefficient because of the poor resolution of aggregates from monomer. SEC resolves species by differences in their hydrodynamic radii. For spherical proteins, the hydrodynamic radius depends approximately on the cubed root of the molecular weight, meaning that increasing the molecular weight 2 -fold will only increase the hydrodynamic radius by $\sim 25 \%$. This size difference is often insufficient to achieve baseline resolution of the monomer and dimer peaks on an analytical SEC column. Worse resolution is typically observed on preparative columns compared with analytical columns because of the large amounts of protein loaded onto these columns. This problem can be exacerbated by dilute protein solutions. To minimize this resolution challenge, the protein loads are concentrated and loaded in multiple cycles to preparative SEC columns. The use of multiple cycles results in a lengthy process, and the resulting pools are fairly dilute.

The use of anion- and cation-exchange chromatography has been demonstrated to be useful at production scale to separate monoclonal antibody monomers from dimers and larger molecular weight species. ${ }^{19}$ Under optimal conditions, the purification of aggregates from monomers using ion exchange chromatography results in solutions containing $<0.5 \%$ aggregate. ${ }^{19}$ These chromatography columns are operated under bind-and-elute conditions, which eliminate some of the issues associated with preparative SEC, including the problems with loading dilute solutions. In addition, the resulting pools are relatively concentrated compared with the pools obtained from SEC.

Filtration membranes are used throughout the purification process to remove impurities, to perform buffer exchange, and to concentrate the protein. ${ }^{20}$ Ultrafiltration/diafiltration (UF/DF) is typically performed to exchange the buffer and to increase the protein concentration in solution. During the UF process, the concentration of protein at the membrane surface may be much higher than that of the bulk solution. This phenomenon may lead to membrane fouling due to the formation of aggregates in the region of locally high concentration. In addition, during the UF/DF process the protein is being continually pumped, with a typical process requiring at least 50 passes through the pump. ${ }^{21,22}$ This mechanical stress may result in an increase in protein aggregation.

Ultrafiltration has also been used to remove aggregates in a monoclonal antibody process. As mentioned in a previous 


\section{The AAPS Journal 2006; 8 (3) Article 66 (http://www.aapsj.org).}

paragraph, the purification of alemtuzumab, the IgG1 monoclonal antibody found in CamPath- $1 \mathrm{H}$, uses SEC chromatography to reduce the substantial levels of irreversible dimers and higher molecular weight oligomers observed, typically on the order of $25 \%$ of the total protein. ${ }^{17,23} \mathrm{~A}$ study was conducted to determine whether ultrafiltration could be more efficient than SEC for aggregate reduction with this protein. ${ }^{23}$ Three membranes were evaluated in the study: $100 \mathrm{kd}$ polyethersulfone (PES), $100 \mathrm{kd}$ polyvinylidene fluoride (PVDF), and $300 \mathrm{kd}$ PES. Selection of pore size is not as trivial as may be anticipated, because the molecular weight cutoff represents a typical pore size and not a limit. The molecular weight of a monoclonal antibody is typically $\sim 150 \mathrm{kd}$, so the molecular weight of a dimer is $\sim 300 \mathrm{kd}$. Thus, the use of a 300-kd membrane may not sufficiently remove the dimers to an acceptable level. Similarly, the 100-kd membrane may have a lower recovery owing to the ability of some monomers to cross the membrane or may be fouled easily because of the presence of a large amount of high molecular weight species. In this study, the effect of different buffer conditions including $\mathrm{pH}$ and ionic strength on the ability of the membranes to separate monomers from aggregates was evaluated. The 100-kd PES membrane quickly fouled, with high resistance and a low flux resulting. No substantial fouling was observed with the 100-kd PVDF or 300-kd PES membranes. Under optimized conditions, the best separation was observed using the 100kd PVDF membrane with final product purity of $93 \%$ monomer and a yield of $85 \%$. The authors predict optimization of pore size and operating parameters could increase the purity to $96 \%$. One additional observation noted in this article regarded the stirring speed used for the study and the effect of shear stress on protein aggregation. Several speeds were tested, and it was observed that stirring speeds of $600 \mathrm{rpm}$ and above resulted in cloudy solutions within the first 2 hours of processing. Clear solutions for greater than 6 hours were obtained by limiting the speed to $300 \mathrm{rpm}$.

\section{Aggregation During Formulation}

As described above, UF/DF is often used to formulate protein therapeutics. It offers the advantage of producing a formulation with high protein concentration and high yield. ${ }^{21}$ During the clinical development for a monoclonal antibody, there was a desire to improve the cost of goods associated with the UF/DF process by changing the conditions under which that process was performed. One key excipient in the formulation was expensive and limited in availability. During the DF portion of the process, 10 diavolumes of the buffer were used to achieve the final solution. This resulted in $90 \%$ of the DF buffer going to waste. Thus, there was a strong desire to remove the expensive excipient from this process because of the large waste and cost. A study was conducted to determine if it was possible to remove this costly excipient from the UF/DF process. The experiment was performed at ambient temperature using a laboratory scale UF/DF system fitted with a diaphragm pump and a $30-k d$ cellulose membrane. Samples were taken at various stages during the UF/DF process and analyzed for protein concentration by UV spectroscopy, turbidity as determined by the absorbance at $350 \mathrm{~nm}$ in a $1-\mathrm{cm}$ path length cell, and percentage aggregate as determined by SEC. It should be noted that using turbidity in this way to monitor particulate formation does not provide absolute numbers on the number of particles present. The light scattering observed using this method will depend on both the size and number of particles. However, it does provide a means of presenting information that is observed visually; solutions that appear more opalescent to the eye produce higher absorbance at $350 \mathrm{~nm}$.

The results from this experiment are displayed in Table 1 . The first sample, Load, represents the pool entering the UF/ DF system and is the same sample for both processes. The normalized turbidity was determined by dividing the Turbidity value by the protein concentration to eliminate any light scattering that results solely as an effect of protein concentration. The next sample described in the table, UF, was taken after the UF process was complete. In each process, the protein was concentrated to $\sim 10 \mathrm{mg} / \mathrm{mL}$ and there was little difference in the product quality observed between the 2 processes. The third sample in each set, DF, was taken after 10 diavolumes of formulation buffer had been exchanged into the protein. For condition 1, the buffer contained the excipient of interest; condition 2 did not contain this excipient. There are essentially no differences in product quality observed between the 2 conditions. The turbidity observed for both conditions was substantially higher than observed in the UF sample, indicating the presence of particulates in the sample. The turbidity further increased for the "Recovered Pool" sample under each condition. This sample was taken after the system was flushed and included any particulates that may have settled during the processing. These particulates resulted from the shear stress experienced from the continual pumping of the protein solution throughout the process. The turbidities observed for the Recovered Pool samples were significantly higher than in the previous pool, with the pool generated by condition 2 showing higher levels of turbidity and slightly increased aggregate compared with that generated by condition 1 . This finding indicates that the excipient used in condition 1 may afford some protection to the protein during the UF/DF operation, although it is possible that the difference in protein concentration contributes to some of the observed differences in quality. The final sample in each set was taken after conditioning of the pools with excipients that were not used during the UF/DF process and after filtration through a $0.22-\mu \mathrm{m}$ membrane. The pools generated under each condition were, once again, in the same buffer and were approximately 
The AAPS Journal 2006; 8 (3) Article 66 (http://www.aapsj.org).

Table 1. Removal of an Excipient From the Diafiltration Buffer Had Minimal Effect on the Product Quality*

\begin{tabular}{|c|c|c|c|c|}
\hline Sample & $\begin{array}{c}\text { Protein Concentration } \\
(\mathrm{mg} / \mathrm{mL})\end{array}$ & $\%$ Aggregate & Turbidity & $\begin{array}{c}\text { Normalized } \\
\text { Turbidity }{ }^{\dagger}\end{array}$ \\
\hline \multicolumn{5}{|c|}{ Condition 1: Excipient present during UF/DF process } \\
\hline Load & 4.2 & 2.7 & 0.015 & 0.004 \\
\hline UF & 10.0 & 2.4 & 0.070 & 0.007 \\
\hline $\mathrm{DF}$ & 7.8 & 2.7 & 0.166 & 0.021 \\
\hline Recovered pool & 9.2 & 3.0 & 0.443 & 0.048 \\
\hline $0.22 \mu \mathrm{m}$ Filtered pool & 9.5 & 2.8 & 0.030 & 0.003 \\
\hline \multicolumn{5}{|c|}{ Condition 2: Excipient removed during UF/DF process } \\
\hline Load & 4.2 & 2.7 & 0.015 & 0.004 \\
\hline UF & 10.2 & 2.4 & 0.059 & 0.006 \\
\hline $\mathrm{DF}$ & 9.5 & 2.9 & 0.200 & 0.021 \\
\hline Recovered pool & 11.1 & 3.3 & 0.587 & 0.053 \\
\hline $0.22 \mu \mathrm{m}$ Filtered pool & 9.4 & 3.0 & 0.036 & 0.004 \\
\hline
\end{tabular}

*UF/DF indicates ultrafiltration/diafiltration.

$\uparrow$ The normalized turbidity was obtained by dividing the Turbidity (absorbance at $350 \mathrm{~nm}$ for an undiluted solution) by the protein concentration.

the same concentration. The turbidity observed in the recovered pools was substantially decreased in the filtered pools and there, once again, was no substantial quality difference between pools. Although visually significant levels of precipitate were observed in this process, their presence did not affect the performance of downstream filters, they could be removed by filtration, and they did not affect long-term stability of the filtered product (data not shown). This experiment supported the removal of the excipient from the process.

\section{Aggregation During Filling}

The effect of protein aggregation on the filling operation may not be given sufficient consideration. As with the purification process, aggregates may be formed during the filling operation. Because there is no further purification after the fill, it is critical that the formation of aggregates is minimized if not prevented completely. For this reason it is essential that compatibility of the protein formulation with the filling equipment is assessed before production.

During clinical development of a monoclonal antibody, it was necessary to transfer the filling operation to a new facility. This facility offered 2 options for the type of filler used. The first type of filler used a rolling diaphragm pump, while the second type of filler used a radial piston pump. The previous manufacturing experience with this protein had used a rolling diaphragm pump with no issues. There was no experience with the piston pump for this protein. One of the concerns with the piston pump was that the protein solution is used to lubricate the piston during the operation, and it was known that this particular protein is sensitive to shear stress.
A study was conducted to evaluate whether the piston pump could be used to fill this protein solution. In this experiment, $1 \mathrm{~L}$ of protein formulation was transferred from 1 reservoir to another at ambient temperature using a piston pump head operating at 1 of 2 speeds, representing the typical and the fastest filling speeds that would be used. A total of 13 transfers occurred between the 2 containers. After each transfer, a sample was taken for analysis by turbidity (absorption at 350 $\mathrm{nm}$ in a 1-cm path length cell) and by light obscuration (Hiac Royco, Grants Pass, OR) to determine the number of small particles. The results of this study are displayed in Figure 2. As the number of pump passes increased, both the turbidity and the number of particles $>2 \mu \mathrm{m} / \mathrm{mL}$ increased substantially. Based on these results and the knowledge that there was no compatibility problem observed during manufacturing with the rolling-diaphragm pump, the decision was made to use only the filler equipped with the rolling-diaphragm pump for this protein.

As mentioned in the Introduction, self-association of proteins may result in the formation of viscous solutions. Consideration of the effect of the viscosity on the filling operation should be given prior to the first production. The ability to accurately fill the viscous solution may be compromised if appropriate process development is not performed. The process development should include investigating the effect on nozzle size and filling speed.

An antibody in clinical development exhibited viscosity that resulted in difficulties in the filling process. The viscosity was found to be highly temperature dependent as shown in Figure 3. Under refrigerated conditions, the viscosity was $\sim 350 \mathrm{cps}$, while at ambient temperature the viscosity was 
The AAPS Journal 2006; 8 (3) Article 66 (http://www.aapsj.org).

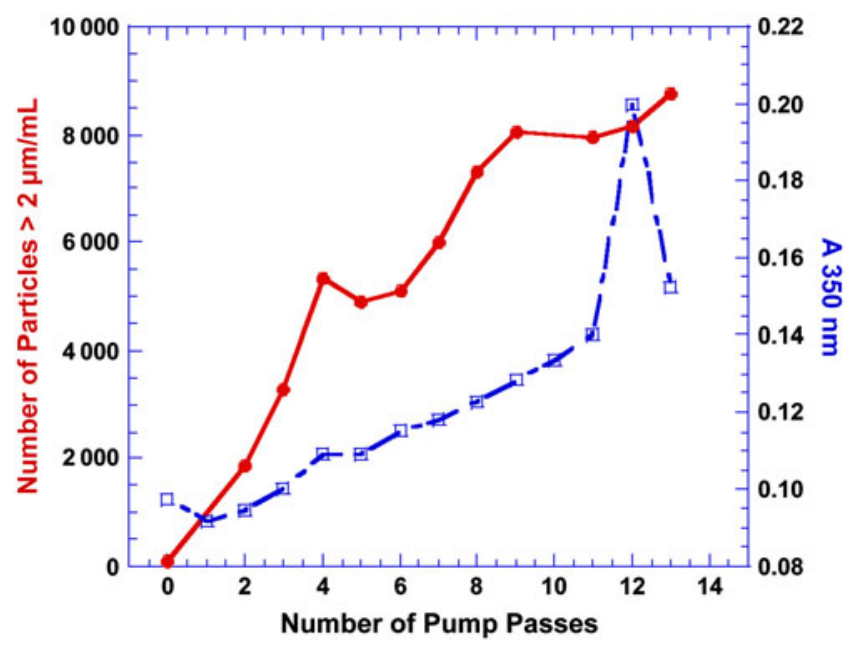

Figure 2. Recirculation of a MAb formulation through a piston pump. The solid line reflects the number of particles $>2 \mathrm{~mm} / \mathrm{mL}$ as determined by Hiac Royco. The dashed line reflects the absorbance at $350 \mathrm{~nm}$ in a $1-\mathrm{cm}$ path length cell and is an indicator of the turbidity of the solution. Both the number of particles and the turbidity increase with an increased number of passes through the pump. The large increase in turbidity observed for the sample taken at pass 12 is believed to result from dried protein shedding from the piston into the solution.

reduced to a more manageable, yet still viscous, 40 to 70 cps. Even by increasing the temperature of the solution being filled, problems were encountered in the initial fill of this product. The formulation was found to drip from the nozzle of the filler resulting in inaccurate fill volumes. During development of the filling process, a combination of different nozzle sizes and filling speeds were tested to maximize the recovery of the protein and to prevent dripping from the

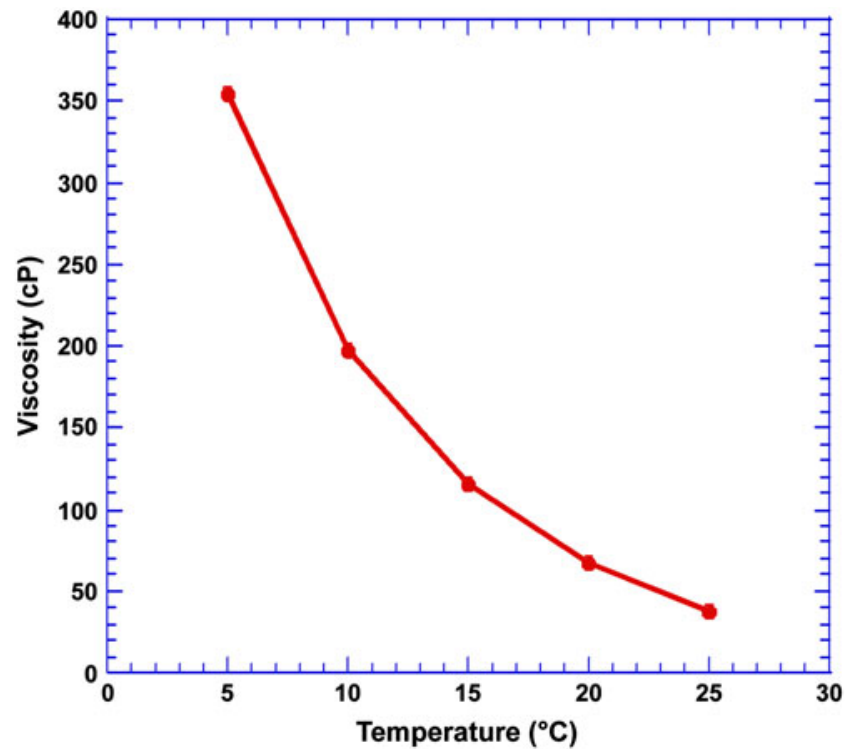

Figure 3. Viscosity of a MAb formulation as a function of temperature. nozzle. This development work resulted in the ability to accurately fill the protein solution.

\section{CONCLUSION}

There is the potential for protein aggregation throughout the manufacturing process of therapeutic proteins. Care should be taken to minimize the aggregates created, particularly in the final filling operation. The purification process may be designed to exploit differences in characteristics of the aggregates compared with the monomers (eg, hydrophobicity, charge, size) to reduce their levels in the final product.

\section{ACKNOWLEDGMENTS}

The authors wish to thank Poonam Mulherkar, Anita Baron, David Overcashier, and Al Stern for technical assistance and Tom Patapoff for helpful discussions.

\section{REFERENCES}

1. US Pharmacopeia. USP/NF General Chapter $<788>$ Particulate Matter in Injections. In: U.S. Pharmacopeia, ed. National Formulary, USP29-NF-24 (suppl 2). Rockville, MD: USP; 2006.

2. Andya JD, Hsu CC, Shire SJ. Mechanisms of aggregate formation and carbohydrate excipient stabilization of lyophilized humanized monoclonal antibody formulations. AAPS PharmSci. 2003;5:E10.

3. Shahrokh Z, Sluzky V, Stratton PR, Eberlein GA, Wang YJ. Disulfide-linked oligomerization of basic fibroblast growth factors: effect of sulfated compounds. In: Formulation and Delivery of Proteins and Peptides, ACS Symposium Series 567. Washington, DC: American Chemical Society; 1994:85-99.

4. Creed D. The photophysics and photochemistry of the near-UV absorbing amino acids. II. Tyrosine and its simple derivatives. Photochem Photobiol. 1984;39:563-575.

5. Giulivi C, Davies KJA. Dityrosine: a marker for oxidatively modified proteins and selective proteolysis. In: Packer L, ed. Methods in Enzymology. vol. 233. New York, NY:

Academic Press; 1994:363-371.

6. Moore JM, Patapoff TW, Cromwell MEM. Kinetics and thermodynamics of dimer formation and dissociation for a recombinant humanized monoclonal antibody to vascular endothelial growth factor. Biochemistry. 1999;38:13960-13967.

7. Liu J, Nguyen MD, Andya JD, Shire SJ. Reversible self-association increases the viscosity of a concentrated monoclonal antibody in aqueous solution. J Pharm Sci. 2005;94:1928-1940.

8. Maislos M, Bialer M, Mead PM, Robbins DC. Pharmacokinetic model of circulating covalent aggregates of insulin. Diabetes. 1988;37:1059-1063.

9. Rosenberg AS. Effects of protein aggregates: an immunologic perspective. AAPS J. 2006;8:E501-E507.

10. Fernández A. What factor drives the fibrillogenic association of $\beta$-sheets? FEBS Lett. 2005;579:6635-6640.

11. Chi EY, Krishnan S, Randolph TW, Carpenter JF. Physical stability of proteins in aqueous solution: mechanism and driving forces in nonnative protein aggregation. Pharm Res. 2003;20:1325-1336. 
The AAPS Journal 2006; 8 (3) Article 66 (http://www.aapsj.org).

12. Zhang YB, Howitt J, McCorkle S, Lawrence P, Springer K, Freimuth P. Protein aggregation during overexpression limited by peptide extensions with large net negative charge. Protein Expr Purif. 2004;36:207-216.

13. Freimuth P, Springer K, Berard C, Hainfeld J, Bewley M, Flanagan J. Coxsackievirus and adenovirus receptor amino-terminal immunoglobulin V-related domain binds adenovirus type 2 and fiber knob from adenovirus type 12. J Virol. 1999;73:1392-1398.

14. Frand AR, Cuozzo JW, Kaiser CA. Pathways for protein disulphide bond formation. Trends Cell Biol. 2000;10:203-210.

15. Zhang W, Czupryn MJ. Free sulfhydryl in recombinant monoclonal antibodies. Biotechnol Prog. 2002;18:509-513.

16. Chaderjian WB, Chin ET, Harris RJ, Etcheverry TM. Effect of copper sulfate on performance of a serum-free $\mathrm{CHO}$ cell culture process and the level of free thiol in the recombinant antibody expressed. Biotechnol Prog. 2005;21:550-553.

17. Phillips J, Drumm A, Harrison P, et al. Manufacture and quality control of CAMPATH-1 antibodies for clinical trials. Cytotherapy. 2001;3:233-242.
18. Ejima D, Yumioka R, Tsumoto K, Arakawa T. Effective elution of antibodies by arginine and arginine derivatives in affinity column chromatography. Anal Biochem. 2005;345:250-257.

19. Ansaldi D, Lester P, inventors. Genentech, Inc., assignee. Separation of polypeptide monomers. US patent 6620918 . September 16, 2003.

20. van Reis R, Zydney A. Membrane separations in biotechnology. Curr Opin Biotechnol. 2001;12:208-211.

21. Harris RJ, Shire SJ, Winter C. Commercial manufacturing scale formulation and analytical characterization of therapeutic recombinant antibodies. Drug Dev Res. 2004;61:137-154.

22. van Reis R, Goodrich EM, Yson CL, Frautschy LN, Dzengeleski S, Lutz H. Linear scale ultrafiltration. Biotechnol Bioeng. 1997;55:737-746.

23. Wan Y, Vasan S, Ghosh R, Hale G, Cui Z. Separation of monoclonal antibody alemtuzumab monomer and dimers using ultrafiltration. Biotechnol Bioeng.

2005;90:422-432. 